\section{The quantum jungle revisited}

The New World of Mr Tompkins

by George Gamow \& Russell Stannard

Cambridge University Press: 1999. 256 pp.

$\mathfrak{1 4}$.95, $\$ 24.95$

\section{Anton Zeilinger}

Have you ever wondered what the twentieth century's two great theories of physics quantum theory and the theory of relativity - really tell us? Every physicist views these theories as providing a major break with the world-view - generally known as classical physics - that existed until the end of the nineteenth century.

The problem with considering these phenomena, and the reason the theories were not discovered earlier, is mainly one of scale relativity theories are concerned with the enormously high speed of light, while quantum mechanics is characterized by Planck's extremely tiny quantum of action. Thus, in general, to directly 'see' relativistic phenomena one must go to speeds far beyond the scale people are familiar with, whereas quantum phenomena can only be seen by analysing changes occurring on extremely small scales.

In 1938, the Russian-born physicist George Gamow, who was at that time living in the United States, invented the elegant idea of considering what phenomena we would experience if the velocity of light was not 300,000 kilometres per second, but instead was the speed achieved by a fast cyclist, and if Planck's quantum of action was large enough for quantum phenomena to be part of everyday experience. Gamow invented "Mr Tompkins", the bank clerk who, curious about modern physics, attended public lectures at the local university. However, having a rather short attention span, he fell asleep during the lectures and dreamt of living in just such a strange world. In his dreams he rode a relativistically flattened bicycle, played billiards where quantum uncertainty added greatly to the excitement of the game, and met tigers that had been quantum-diffracted by the trees in the jungle.

Gamow's book was published in 1939 as Mr Tompkins in Wonderland, with a sequel, Mr Tompkins Explores the Atom, in 1944. The two books appeared together as Mr Tompkins in Paperback (Cambridge University Press) in 1965.

While these charming stories have been a source of great pleasure to many physicists, some are unfortunately becoming outdated, although they remain interesting historical documents. Given the developments of physics in the second half of the twentieth century, the physicist Russell Stannard, a popular-science writer perhaps best known for his Uncle Albert children's trilogy involving the adventures of Einstein, undertook the task of
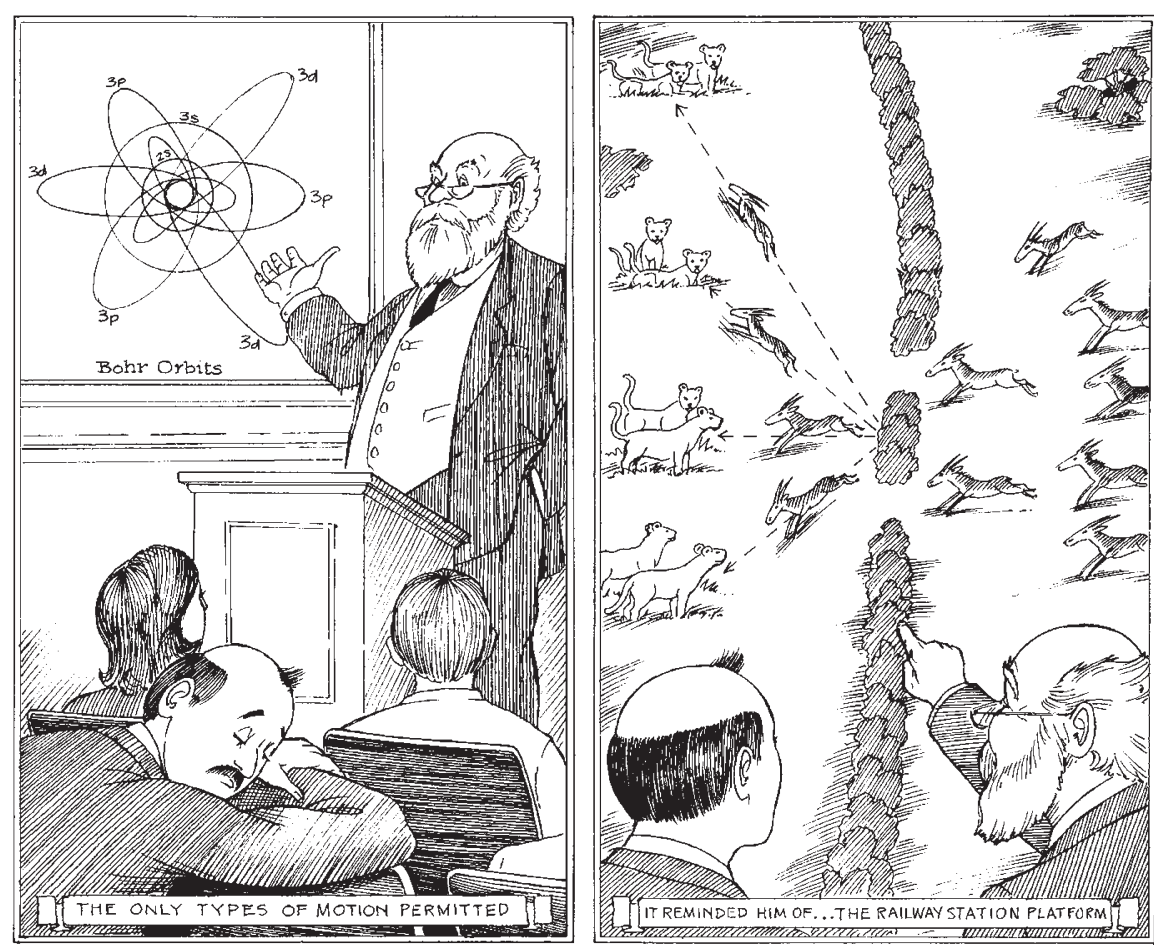

Quantum dreaming: Mr Tompkins' short attention span delivered him to a strange other world.

rewriting some of the stories and adding new material. Particularly useful are his additions on cosmology, which include black holes and the modern unified theory of particle physics. There is a beautiful explanation of the $\mathrm{SU}(3)$ representation, which explains how elementary particles can be understood on the basis of deeper symmetries, and how quarks are able to synthesize all the new particles observed in high-energy physics.

Certainly the most difficult task, both in Gamow's time and today, is to explain quantum mechanics to the general public. This is because its concepts are completely counterintuitive, and because even today's physicists disagree about what it means philosophically. So it is not surprising that Gamow's original presentation may not be totally satisfactory today.

Stannard has rewritten a significant part of Gamow's original chapters on quantum mechanics, but not always in a way that makes them clearer. Whereas Gamow began his explanation using an analogy to billiard balls, without explaining clearly why the concept of quantum mechanics is so strange and thus leaving the reader pretty much in the dark, Stannard starts by describing how we cannot obtain a precise measurement for the track of an elementary particle because of the unavoidable disturbance caused by the measuring process. Although the discussion is valid, it might leave the reader believing that, while we cannot precisely determine, say, the properties of an elementary particle because of the clumsiness of our equipment, the particle may still have well-defined properties; this position is untenable today.

Also, in his discussion of the 'quantum jungle', Stannard introduces the famous double-slit diffraction experiment using the analogy of gazelles which, escaping from an attacking lion through two gaps in a hedge, quantum-diffract and end up in front of lionesses waiting at the positions where the interference maxima are to be expected. This analogy, attractive though it is, is inaccurate in that Stannard says the gazelles have no choice other than to act as they do because their dynamics is governed by quantum mechanics. This is incorrect, because the gazelles merely need to approach the 'double-slit' hedge at a slightly different angle, so that their interference maxima lie between the waiting lionesses, in order to escape.

Actually, from the point of view of modern physics, it is completely impossible to create a detailed visual description of what happens in a quantum experiment between the preparation of the system and the final observation. Therefore, even Gamow's drawings showing probability clouds or smeared-out tigers should be taken with a substantial grain of salt. Given their invalidity in principle, it might have been better to retain Gamow's original figures and thus, through their historic quaintness, imply that the conceptual picture is outdated, rather than replace them with new ones which still have their severe limitations.

That said, although presenting quantum mechanics accurately to a broad lay readership will certainly remain a challenge for some time, The New World of Mr Tompkins is a lovely book which I am sure everyone interested in modern physics, from the age of 11 upwards, will enjoy enormously.

Anton Zeilinger is at the Institut für

Experimentalphysik, University of Vienna,

Boltzmanngasse 5, 1090 Vienna, Austria. 